\title{
The Impact of Standardized Decision Support on Syndromic Surveillance in Alberta
}

\author{
Laura Rivera ${ }^{1}$, Faiza Habib ${ }^{3}$, Ye Li ${ }^{1}$,, Rita K. Biel ${ }^{3}$, Rachel Savage ${ }^{2}$, Natasha Crowcroft ${ }^{1}$, \\ ${ }^{2}$, Laura Rosella ${ }^{2,1}$, Shelly Bolotin ${ }^{1,2}$, David Strong ${ }^{3,4}$, Christopher Sikora ${ }^{3,5}$ and lan \\ Johnson ${ }^{\star 1,2}$
}

${ }^{1}$ Public Health Ontario, Toronto, ON, Canada; ${ }^{2}$ University of Toronto, Toronto, ON, Canada; ${ }^{3}$ Alberta Health Services, Edmonton, AB, Canada; ${ }^{4}$ University of Calgary, Calgray, AB, Canada; ${ }^{5}$ University of Alberta, Edmonton, AB, Canada

\section{Objective}

The objective of this study was to carry out a mixed-methods evaluation of the ability of standardized supports to improve the usefulness of school absenteeism syndromic surveillance for public health in Alberta.

\section{Introduction}

Syndromic surveillance uses pre-diagnostic data to inform communicable disease prevention and control. Among health zones in the province of Alberta, Canada, practices employed by public health when using elementary school illness-cause absenteeism data vary widely.

\section{Methods}

The Alberta Real Time Surveillance System Network (ARTSSN) collects, analyzes and reports on school absenteeism data for all schools in participating health zones across Alberta. The two largest health zones in Alberta, Edmonton Zone and Calgary Zone, participate in ARTSSN and further agreed to participate in a study where one health zone was randomly allocated (flip of a coin) were randomized to receive the standardized supports intervention, or to continue with their regular practice. The intervention consisted of (1) a cumulative sum aberration detection algorithm applied to the ARTSSN data and (2) a protocol outlining how to investigate and follow up on aberrant events. The zone receiving the intervention additionally continued with their regular practices to ensure they could meet their required public health responsibilities. Staff in both intervention and control zones used electronic data collection forms, known as "logbooks" to document and track alerts received, any subsequent investigations done, and any public health action as a result of the alert. At the end of the data collection period, semi-structured interviews were conducted to understand more fully their alert investigation method, rationale for public health action, and their uptake of the study intervention. Frequencies of logbook elements were generated for numeric or categorical data to compare descriptive statistics between intervention and control groups, and logbook free-text elements and qualitative data collected through semi-structured interviews were analyzed thematically. The analysis was performed using Excel, and SAS statistical software.

\section{Results}

The Edmonton (intervention) Zone relied heavily on the regular ARTSSN alerts and study generated alerts. Between February 2014 and February 2015, the intervention zone investigated 246 alerts. In comparison, the Calgary (control) Zone relied on public health nurses communicating regularly with school principals to detect outbreaks in the schools. During the same time period, the control zone investigated 20 alerts. The intervention health zone resulted in the detection of 19 outbreaks, while the control zone resulted in 16 outbreaks detected. In terms of public health actions, there were 39 instances where the intervention health unit provided advice on environmental cleaning as a method of infection control. These actions increased in the second year of the study after 4 hours per week of dedicated nursing time were assigned. While many of the outbreaks in the control health zone were monitored, follow-up actions such as advice on environmental cleaning were not reported.

\section{Conclusions}

These study findings suggest that using automated processes to analyze school absenteeism data results in more frequent alerts than traditional systems relying on principal-nurse interactions. The response to the syndromic surveillance was augmented by the presence of standardized processes, particularly with respect to public health actions such as advice on environmental cleaning, and frequent hand washing. This increase in response only occurred once sufficient human resources were provided to investigate the alerts.

\section{Keywords}

Syndromic surveillance; public health; school absenteeism; Evaluation

\section{*Ian Johnson}

E-mail: ian.johnson@oahpp.ca 